\title{
Atopic dermatitis and filaggrin deficiency lead to characteristic shifts of the skin microbiome
}

\author{
Hansjörg Baurecht, Elke Rodríguez, Frederieke Thielking, Stephan Weidinger
}

Dartment of Dermatology, Allergology and Venerology, University Hospital Schleswig-Holstein, Campus Kiel, Kiel, Germany

\section{Background}

- Genomic approaches revealed characteristic site-specifity of skin bacterial community structures.

- In children with atopic dermatitis $(\mathrm{AD})$ characteristic shifts of the skin microbiome at creases were described and has been postulated to mirror the disturbed skin barrier function and/or cutaneous inflammation.

Are altered cutaneous bacterial community structures in $\mathrm{AD}$ restricted to predilection sites or a general feature?

Have abnormalities of epidermal barrier function and eczematous affection an impact on the skin microbiome?

A
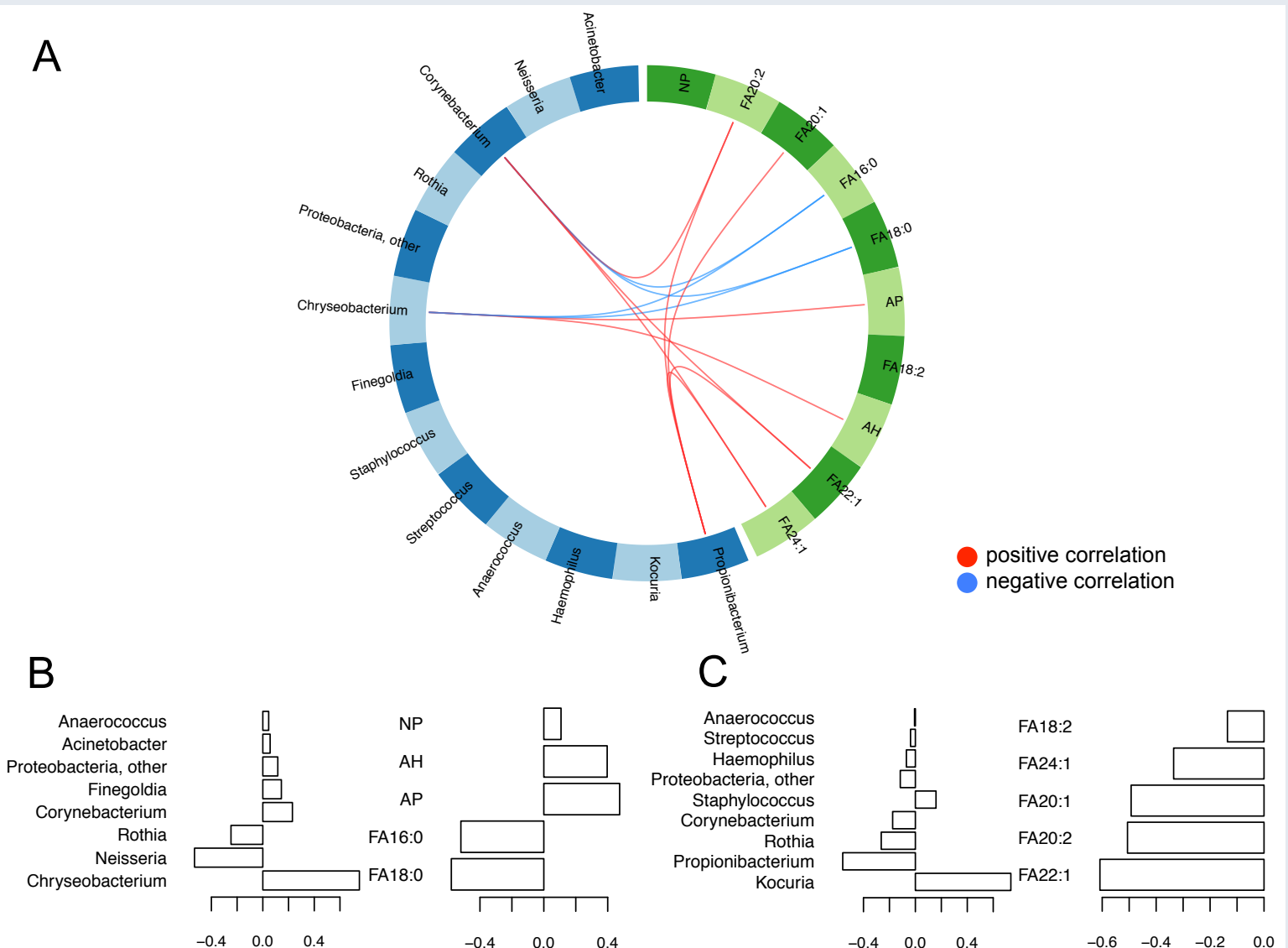

Fig. 2: Impact of epidermal lipid composition on bacterial community composition. (A) Circos plot shows strongly correlated ( $r>0.6)$ features of the skin lipids \& microbiome identified by sparse canonical correlation analysis (sCCA). (B-C) Coefficients weights of genera \& lipids selected by sCCA on the first (B) and second (C) components of each omics level.
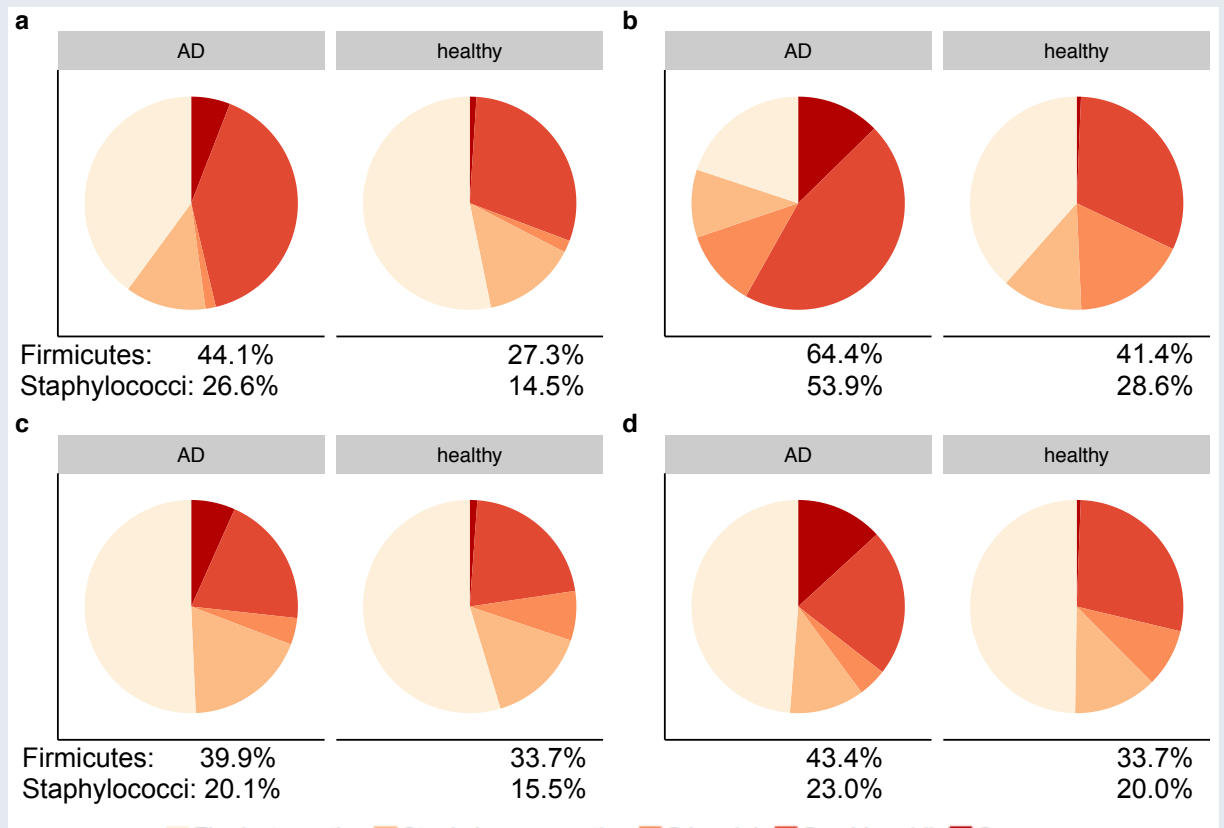

Firmicutes, other

Fig. 3: Effect of AD on the skin microbiome. Mean bacterial abundance of prevalent staphylococi among Firmicutes. (a) forehead, (b) antecubital fossa, (c) extensor forearm, (d) volar forearm. Below each sub-figure the mean relative abundance of Firmicutes and Staphylococci are presented.

\section{Conclusion}

- Stratum corneum integrity and epidermal lipid composition impact skin microbiome composition.

- AD characterized by shifts of the skin microbiome also at non-affected sites, but in particular at sites of predilection and eczematous affection.

- Skin inflammation overlays locoregional differences in microbiome composition.
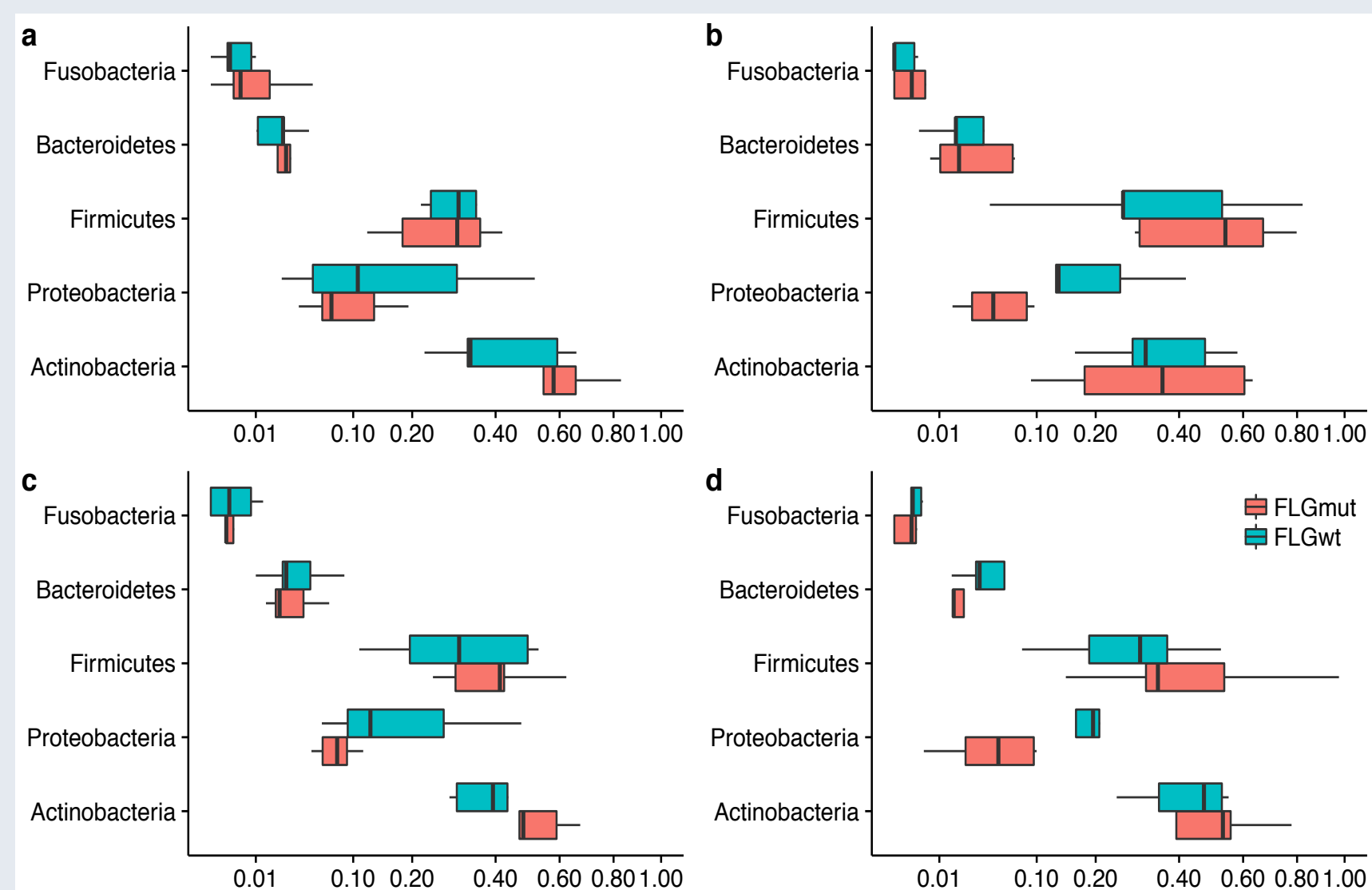

Fig. 1: Effect of $F \boldsymbol{L} G$ deficiency on the skin microbiome. Bacterial abundance of prevalent phyla in healthy individuals. (a) forehead, (b) antecubital fossa, (c) extensor forearm, (d) volar forearm.

\section{Methods}

- 10 AD patients, 10 controls matched for age, sex and FLG mutation status (FLGmut/FLGwt).

- Skin microbiome determined by $16 \mathrm{~S}$ rRNA sequencing at antecubital fossa (Af), forehead (Fh), volar and extensor forearm (Vf, Ef) as well as in acute and chronic AD lesions.

- Sampling sites characterized for skin physiology parameters including TEWL, pH and chromatography-based lipid profiles.

\section{Results and discussion}

- FLG deficient skin showed distinct microbiome compostion (Fig. 1) partly resembling the AD-related pattern.

- Long-chain unsaturated free fatty acids (FFAs) strongly correlate with bacterial composition, in particular Propionibacteria \& Corynebacteria abundance (Fig. 2).

- AD shows a distinct bacterial community structure with increased and altered staphylococci abundance.

- Progressive shift of staphylococcal species composition from nonlesional skin to acute and chronic lesions.

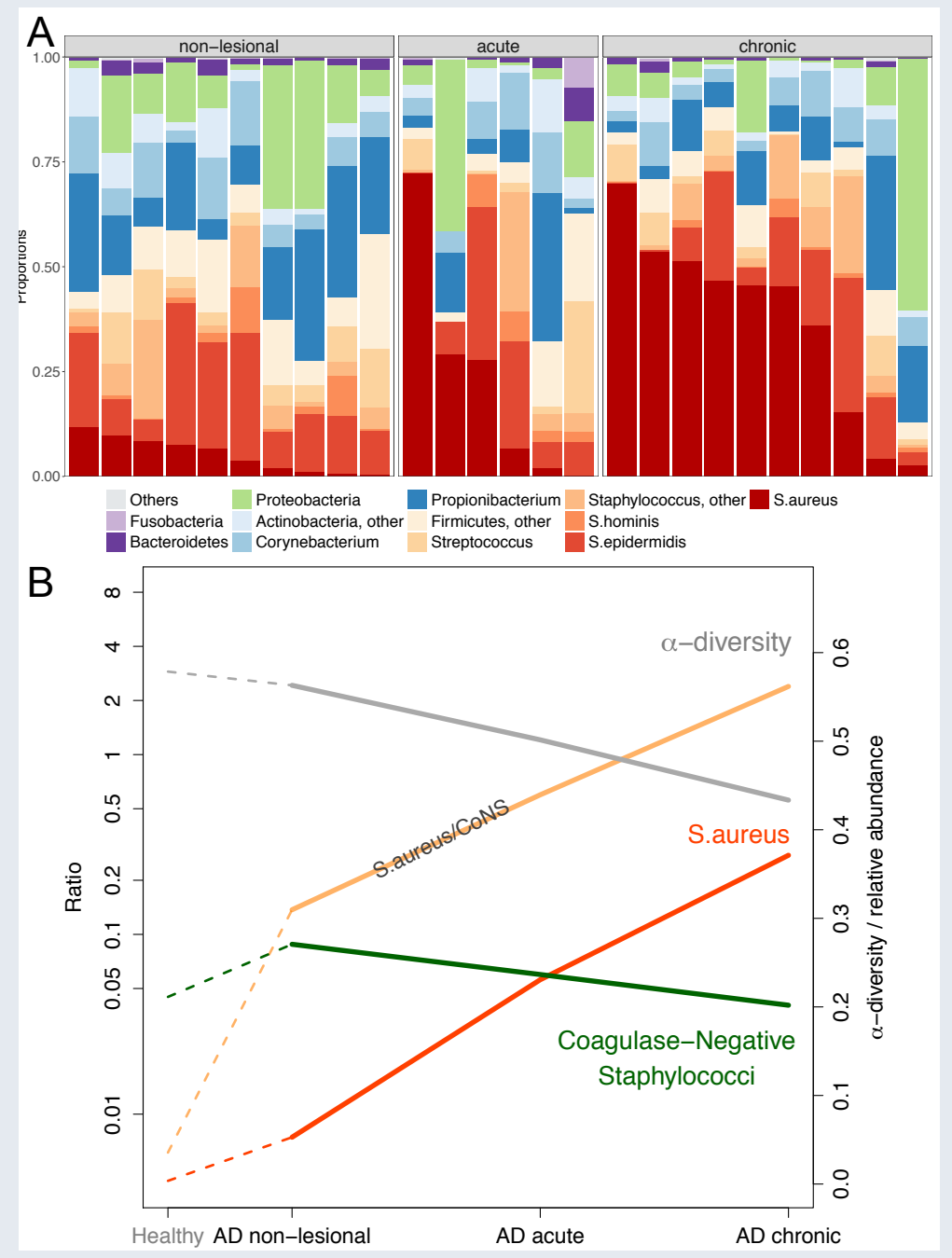

Fig. 4: Change of the skin microbiome from non-lesional to acute and chronic lesional skin. (A) Relative abundance of major phyla, genera and Staphylococcus species in non-lesional, acute and chronic AD skin.

(B) Red \& green lines indicate $\mathrm{S}$. aureus \& Coagulase-Negative Staphylococci (CoNS) abundances with corresponding $y$-axis on the right hand side. Skin-colored line depicts $\mathrm{S}$. aureus/CoNS ratio with log-scaled yaxis on the left hand side. Gray line shows the normalized $\alpha$-diversity with the corresponding $y$-axis on the right hand side. Dotted lines depict the connections to the respective mean value of healthy individualsSkincolored line depicts $\mathrm{S}$. aureus 